\title{
ON OBLIQUE WAVES FORCING BY A POROUS CYLINDRICAL WALL
}

\author{
M. S. FALTAS \\ Department of Mathematics \\ University of Bahrain \\ STATE OF BAHRAIN
}

(Received February 24, 1994 and in revised form October 21,1994)

\begin{abstract}
The problem of oblique cylindrical linearized wave motion is considered for a fluid of infinite depth or finite constant depth in the presence of an impermeable cylindrical wall and coaxial porous wall immersed vertically in the fluid The motion is generated once by the oscillations, which are periodic in time and in $\theta$-direction, of the impermeable wall and next by the porous wall. The velocity potentials have been found in closed forms in the different regions of the fluid and then calculating the hydrodynamic pressure distribution on the porous wall and the profile of the free surface. The scattering problem of oblique waves is then considered A wave trapping phenomenon is investigated. Numerical results are given to the case of radial incident waves and the case when the angle of incident waves is $30^{\circ}$ to the radial direction.
\end{abstract}

KEY WORDS AND PHRASES. Surface Waves, Porous Medium 1991 AMS SUBJECT CLASSIFICATION CODE. 76B 15

\section{INTRODUCTION.}

The scattering of surface waves obliquely incident on partially immersed or completely submerged vertical barriers and plates in infinite fluid were investigated by Faulkner [1,2], Jarvis and Taylor [3], Evans and Morris [4], Rhodes-Robinson [5] and Mandal and Goswami [6]. Levine [7] considered the scattering of surface waves obliquely incident on a submerged circular cylinder. The problem of scattering of oblique waves by a shallow draft cylinder at the free surface was solved by Garrison [8]. Subsequently, Bai [9] studied the more general problem of scattering of oblique waves by a partially immersed cylinder. In all of such works the immersed bodies are assumed to be impermeable. Chwang [10] considered a porous wavemaker oscillating normally to its plane with a constant amplitude. In his linearized analysis, the wavemaker is located in the middle of an infinitely long channel with constant depth. Chwang and $\mathrm{Li} \mathrm{[11]} \mathrm{applied} \mathrm{the} \mathrm{linearized} \mathrm{porous} \mathrm{wavemaker} \mathrm{method} \mathrm{developed} \mathrm{in} \mathrm{[10]} \mathrm{to}$ investigate the small amplitude surface waves produced by a piston-type porous wavemaker near the end of a semi-infinitely long channel of constant depth. Chwang and Dong [12] studied the problem of reflection and transmission of small amplitude surface waves by a vertical porous plate fixed near the end of a semi-infinitely long open channel of constant depth. Gorgui and Faltas [13] extended Chwang's work to include the study of wave motion for a fluid of infinite horizontal extend and of infinite or finite constant depth in the presence of an impermeable plate and a porous wall immersed in the fluid parallel to each other. The waves are generated by arbitrary prescribed horizontal oscillations performed by the impermeable plate or the porous wall. 
In the present paper we investigate the case of oblique cylindrical wave motion in fluids of infinite depth or finite constant depth The linearized theory for waves of small amplitude is used to analyze the forced motion in fluids bounded internally by an impermeable vertical circular cylinder surrounded by a coaxial cylindrical porous wall The waves are generated by arbitrary prescribed oscillations, which are periodic in time and in $\theta$-direction, first performed by the impermeable wall and later by the porous wall It is assumed that the pores of the wall are of such nature as to allow the application of Darcy's law that the fluid velocity normal to the wall is linearly proportional to the difference in pressure between its two sides The method of separation of variables is applied to find analytic solution in closed forms for the linearized boundary value problem in the different regions of the fluid The results of Sections 3 and 4 are used to find the reflection coefficient of the reflected waves due to the scattering of time and $\theta$ periodic waves incident with angle $\beta$ to the radial direction In the last section numerical results are presented for the two cases of radial oscillations and the case of $\beta=30^{\circ}$

\section{BOUNDARY VALUE PROBLEM}

We consider here the excitation of gravity waves on the surface of a fluid by an impermeable vertical cylindrical wall of circular cross-section of radius $a$ that performs oscillations which are periodic in time and in $\theta$-direction. A coaxial cylindrical porous wall of circular cross-section of radius $b(>a)$ is fixed in the fluid (see Fig. 1). Let $(r, \theta, y)$ be cylindrical coordinates with the origin 0 in the undisturbed free surface such that $0 y$ pointing down into the fluid coinciding with the axis of the impermeable and porous walls.

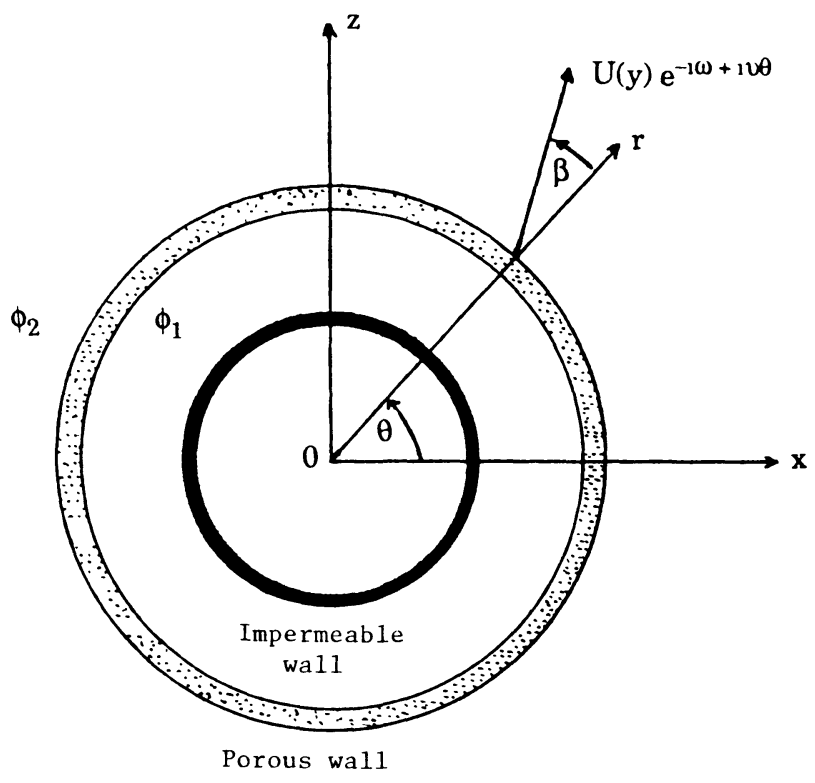

Fig. 1: Schematic diagram of a horizontal cross-section of the physical problem.

Let the velocity of the impermeable wall at time $t$ is $U(y) \exp (-i \omega t+i v \theta)$, where $v=\sin \beta, \beta$ is the angle that the produced train of waves makes with the radial direction and $U(y)$ is a complex valued and suitably limited. The resulting motion is therefore time and $\theta$ harmonics with the same $\omega$ and $v$ as of the impermeable wall. 
We assume that the fluid is incompressible and inviscid and that the motion originates from rest, by virtue of which there exist velocity potentials $\phi_{j}(r, \theta, y ; t)$ such that

$$
\phi_{J}(r, \theta, y ; t)=\operatorname{Re}\left[\phi_{J}(r, y) \exp (-i \omega t+\imath v \theta)\right]
$$

where the subscripts $\jmath=1,2$ refer to the regions $a<r<b$ and $r>b$ respectively Also the motion is assumed small so that the linearization is permissible. We consider here first the case when the fluid is of infinite depth The functions $\phi_{J}(r, y)$ satisfy

$$
\left[\frac{\partial^{2}}{\partial r^{2}}+\frac{1}{r} \frac{\partial}{\partial r}+\frac{\partial^{2}}{\partial y^{2}}-\frac{v^{2}}{r^{2}}\right] \phi_{J}=0, \quad y>0,
$$

The linearized free surface condition is

$$
K \phi_{\jmath}+\frac{\partial}{\partial y} \phi_{\jmath}=0, \quad \text { on } \quad y=0
$$

where $K=\frac{\omega^{2}}{g}$ and $g$ is the gravitational constant.

On the impermeable wall

$$
\frac{\partial}{\partial r} \phi_{1}=U(y), \quad \text { on } \quad r=a
$$

and on the porous wall

$$
\frac{\partial}{\partial r} \phi_{1}=\frac{\partial}{\partial r} \phi_{2}, \quad \text { on } \quad r=b .
$$

We shall also assume that the porous wall is made of material with very fine pores. Thus according to Taylor's assumption [14] we have

$$
\frac{\partial}{\partial r} \phi_{\jmath}=\frac{d}{\mu}\left(p_{1}-p_{2}\right)=i G\left(\phi_{1}-\phi_{2}\right), \quad \text { on } \quad r=b,
$$

where $g=\rho \omega d / \mu, \mu$ is the dynamic viscosity, $\rho$ is the constant density of the fluid and $d$ is a coefficient which has the dimension of length. It should be noted here if the porous flow through the wall is significant, condition (2.5) may not be accurate enough. Hence we should confine our investigation to porous walls with fine pores. Finally we have the condition for no motion at infinite depth,

$$
\nabla \phi_{j} \rightarrow 0 \quad \text { as } \quad y \rightarrow \infty
$$

and the radiation condition for the outgoing waves

$$
\phi_{2} \rightarrow C H_{v}^{(1)}(K r) e^{-K y} \quad \text { as } \quad r \rightarrow \infty
$$

where $C$ is multiple constant and $H_{v}^{(1)}(z)$ is Hankel's Bessel function of third kind of order $v$. The parameter $G$ is a measure of the porous effect. $G=0$ means the wall is impermeable, while as $G$ approaches infinity the wall becomes completely permeable to the fluid.

\section{SOLUTION}

Using the method of separation of variables and superposing basic solutions of Laplace's equation (2.1) appropriate to the present problem, let

$$
\begin{aligned}
\phi_{1}(r, y)= & \int_{0}^{\infty}\left[A(k) I_{v}(k r)+B(k) K_{v}(k r)\right] f(k, y) d k \\
& +\left[\alpha J_{v}(K r)+\beta H_{v}^{(1)}(K r)\right] e^{-K y}
\end{aligned}
$$




$$
\phi_{2}(r, y)=\int_{0}^{\infty} C(k) K_{v}(k r) f(k, y) d k+\gamma H_{v}^{(1)}(K r) e^{-K^{y} y}
$$

where $f(k, y)=k \cos k y-K \sin k y$, and, as usual, $\left(J_{v}(z), Y_{v}(z)\right)$ and $\left(I_{v}(z), K_{v}(z)\right)$ are respectively the Bessel and modified Bessel functions of $v$-th order

These satisfy (2 2), (26) and (2 7) Conditions (24) and (2 5) give $A, B$ in terms of $C$ and $\alpha, \beta$ in terms of $\gamma$ as

$$
\begin{array}{rlrl}
A & =-\frac{b}{i G} K_{v}^{\prime 2}(k b) C, & B & =\frac{1}{i G}\left[i G+b K_{v}^{\prime}(k b) I_{v}^{\prime}(k b)\right] C, \\
\alpha & =-\frac{\pi b}{2 G}\left[H_{v}^{(1) \prime}(K b)\right]^{2} \gamma, \quad \beta=\frac{1}{G}\left[G+\frac{\pi b}{2} J_{v}^{\prime}(K b) H_{v}^{(1) \prime}(K b)\right] \gamma,
\end{array}
$$

where' denotes differentiation with respect to $r$.

From (3 1), (3 3), (2.3) we get

$$
U(y)=\frac{\pi i}{2 G} e^{\pi v v / 2} \int_{0}^{\infty} C(k) \triangle(i k) f(k, y) d k+\frac{\gamma}{G} \triangle(K) e^{-K y}
$$

in which

$$
\triangle(K)=G H_{v}^{(1) \prime}(K a)+M(K) H_{v}^{(1) \prime}(K b)
$$

where we have used the Wronskian relations

$$
W\left[I_{v}(z), K_{v}(z)\right]=-\frac{1}{z} \quad \text { and } \quad W\left[H_{v}^{(1)}(z), J_{v}(z)\right]=\frac{2 i}{\pi z}
$$

in (3.5)

$$
M(K)=\frac{1}{2} \pi b\left[H_{v}^{(1) \prime}(K a) J_{v}^{\prime}(K b)-H_{v}^{(1) \prime}(K b) J_{v}^{\prime}(K a)\right]
$$

Multiplying (3.4) by $e^{-K y}$ and integrating with respect to $y$ from 0 to $\infty$ we get

$$
\gamma=\frac{2 \pi K A G}{\triangle(K)}, \quad \text { where } \quad A=-\frac{1}{\pi K} \int_{0}^{\infty} U(y) e^{-K y} d y .
$$

But $U(y)$ has the unique expansion

$$
U(y)=-2 \int_{0}^{\infty} \frac{k a(k)}{k^{2}+K^{2}} f(k, y) d k-2 \pi K A e^{-K y},
$$

where

$$
a(k)=-\frac{1}{\pi k} \int_{0}^{\infty} U(y) f(k, y) d y,
$$

which can be easily proved by a straightforward application of the Fourier sine integral of $U(y)$.

Comparing (3.4) and (3.6) we obtain

$$
C(k)=\frac{4 i e^{-i \pi v / 2} k G a(k)}{\pi\left(k^{2}+K^{2}\right) \triangle(i k)} .
$$

Hence 


$$
\begin{aligned}
\phi_{1}= & \frac{4}{\pi} e^{-\imath \pi v / 2} \int_{0}^{\infty} \frac{k a(k) f(k, y)}{\left(k^{2}+K^{2}\right) \triangle(i k)}\left[\left[i G+b K_{v}^{\prime}(k b) I_{v}^{\prime}(k b)\right] K_{v}(k r)\right. \\
& \left.-b K_{v}^{\prime 2}(k b) I_{v}(k r)\right] d k-\frac{2 \pi K A}{\triangle(K)}\left[\left[G+\frac{1}{2} \pi b J_{v}^{\prime}(K b) H_{v}^{(1) \prime}(K b)\right] H_{v}^{(1)}(K r)\right. \\
& \left.-\frac{1}{2} \pi b\left[H_{v}^{(1) \prime}(K b)\right]^{2} J_{v}(K r)\right] e^{-K y}, \\
\phi_{2}= & \frac{4}{\pi} i e^{-\imath \pi v / 2} \int_{0}^{\infty} \frac{k G a(k) f(k, y)}{\left(k^{2}+K^{2}\right) \triangle(i k)} K_{v}(k r) d k-\frac{2 \pi K A G}{\triangle(K)} H_{v}^{(1)}(K r) e^{-K y}
\end{aligned}
$$

The hydrodynamic pressure distribution on the porous wall $(r=b)$ is

$$
P=\frac{4}{\pi} i \rho \omega e^{-\imath \pi v / 2} \int_{0}^{\infty} \frac{k a(k) f(k, y)}{\left(k^{2}+K^{2}\right) \triangle(i k)} K_{v}^{\prime}(k b) d k-\rho \omega \frac{2 \pi K A}{\triangle(K)} H_{v}^{(1) \prime}(K r) e^{-K y},
$$

and the free surface elevation is

$$
\begin{aligned}
\omega \eta_{1}(r)= & -\frac{4}{\pi} i K e^{-\imath \pi v / 2} \int_{0}^{\infty} \frac{k^{2} a(k)}{\left(k^{2}+K^{2}\right) \triangle(i k)}\left[\left[i G+b K_{v}^{\prime}(k b)\right] K_{v}(k r)\right. \\
& \left.-b K_{v}^{\prime 2}(k b) I_{v}(k r)\right] d k+i \frac{2 \pi K^{2} A}{\triangle(K)}\left[\left[G+\frac{1}{2} \pi b J_{v}^{\prime}(K b) H_{v}^{(1) \prime}(K b)\right] H_{v}^{(1)}(K r)\right. \\
& \left.-\frac{1}{2} \pi b\left[H_{v}^{(1) \prime}(K b)\right]^{2} J_{v}(K r)\right] \\
\omega \eta_{2}(r)= & \frac{4}{\pi} K G e^{-\imath \pi v / 2} \int_{0}^{\infty} \frac{k^{2} a(k)}{\left(k^{2}+K^{2}\right) \triangle(i k)} K_{v}(k r) d k+i G \frac{2 \pi K^{2} A}{\triangle(K)} H_{v}^{(1)}(K r)
\end{aligned}
$$

The second term on the right hand side of equation (3.11) represents the outgoing waves transmitted through the porous cylinder.

When the porous wall $(r=b)$ is completely permeable i.e. $G \rightarrow \infty$, the velocity potential in the region $r>a$ is

$$
-2 \int_{0}^{\infty} \frac{k a(k) f(k, y)}{k^{2}+K^{2}} \frac{K_{v}(k r)}{K_{v}^{\prime}(k a)} d k-2 \pi K A \frac{H_{v}^{(1)}(K r)}{H_{v}^{(1) \prime}(K a)} e^{-K y}
$$

Also when the porous wall at $(r=b)$ becomes impermeable, $G=0$, the results (3.7), (3.8) reduce to

$$
\begin{aligned}
\phi_{1}= & 2 \int_{0}^{\infty} \frac{k a(k) f(k, y)}{k^{2}+K^{2}} \frac{K_{v}^{\prime}(k b) I_{v}(k r)-I_{v}^{\prime}(k b) K_{v}(k r)}{K_{v}^{\prime}(k a) I_{v}^{\prime}(k b)-K_{v}^{\prime}(k b) I_{v}^{\prime}(k a)} d k \\
& +2 K A \frac{H_{v}^{(1) \prime}(K b) J_{v}(K r)-J_{v}^{\prime}(K b) H_{v}^{(1)}(K r)}{H_{v}^{(1) \prime}(K a) J_{v}^{\prime}(K b)-H_{v}^{(1) \prime}(K b) J_{v}^{\prime}(K a)} e^{-K y}, \\
\phi_{2}= & 0 .
\end{aligned}
$$

This solution is valid only when the quantity

$$
Y_{v}^{\prime}(K a) J_{v}^{\prime}(K b)-Y_{v}^{\prime}(K b) J_{v}^{\prime}(K a)
$$

is different from zero. However, it indicates that when this quantity vanishes, resonance occurs and linearized theory for small motion cannot be applied.

In the particular case when $U(y)=V e^{-K y}$, where $V$ is a real constant, we have 


$$
\begin{aligned}
\phi_{2}= & \frac{V}{\triangle(K)}\left[\left[G+\frac{1}{2} \pi b J_{v}^{\prime}(K b) H_{v}^{(1) \prime}(K b)\right] H_{v}^{(1)}(K r)-\frac{1}{2} \pi b\left[H_{v}^{(1) \prime}(K b)\right]^{2}\right. \\
& \left.\times H_{v}^{(1)}(K r)\right] e^{-K y} \\
\phi_{2}= & \frac{G V}{\triangle(K)} H_{v}^{(1)}(K r) e^{-K y},
\end{aligned}
$$

The distribution of pressure on $r=b$ is

$$
P=\frac{\rho \omega V}{\triangle(K)} H_{v}^{(1) \prime}(K b) e^{-K y} .
$$

\section{THE FINITE DEPTH CASE}

Now we consider the case of finite depth $h$. Using the same notation and coordinates, the complex potentials $\phi_{j}, j=1,2$, for the motion in the fluid regions $\left.a<r<b, r\right\rangle b$ are the solutions of the boundary value problem stated in Section 2 with conditions (2.6), (2.7) replaced by

$$
\begin{gathered}
\frac{\partial}{\partial y} \phi_{\jmath}=0 \quad \text { on } y=h, \\
\phi_{2} \rightarrow C H_{v}^{(1)}\left(k_{0} r\right) \cosh k_{0}(h-y) \quad \text { as } \quad r \rightarrow \infty
\end{gathered}
$$

when $C$ is a constant multiple, $k_{0}$ is the real positive root of

$$
k \sinh k h-K \cosh k h=0 .
$$

The method of separation of variables can also be used here to get solutions for the equations (2.1) that satisfy (2.2), (4.1) and (2.2), (4.1), (4.2). Let then

$$
\begin{gathered}
\phi_{1}(r, y)=\sum_{n=1}^{\infty}\left[A_{n} I_{v}\left(k_{n} r\right)+B_{n} K_{v}\left(k_{n} r\right)\right] \cos k_{n}(h-y) \\
+\left[\alpha J_{v}\left(k_{0} r\right)+\beta H_{v}^{(1) \prime}\left(k_{0} r\right)\right] \cosh k_{0}(h-y), \\
\phi_{2}(r, y)=\sum_{n=1}^{\infty} C_{n} K_{v}\left(k_{n} r\right) \cos k_{n}(h-y)+H_{v}^{(1)}\left(k_{0} r\right) \cosh k_{n}(h-y),
\end{gathered}
$$

where $k_{n}$ are the real positive roots of

$$
k \sin k h+K \cos k h=0 .
$$

The remaining conditions (2.3)-(2.5) are satisfied if

$$
U(y)=\frac{\pi i}{2 G} e^{-\imath \pi v / 2} \sum_{n=1}^{\infty} C_{n} \Delta\left(i k_{n}\right) \cos k_{n}(h-y)+\frac{\gamma}{G} \Delta\left(k_{0}\right) \cosh k_{0}(h-y)
$$

since the eigenfunctions $\cosh k_{0}(h-y)$ and $\cos k_{n}(h-y)$ are orthogonal over the inteval $(0, h)$, we obtain the constants as

$$
C_{n}=8 i e^{-\imath \pi v / 2} \frac{k_{n} a_{n} G \cosh k_{n} h}{\delta_{n} \triangle\left(i k_{n}\right)}, \quad \gamma=-4 \pi \frac{k_{0} a_{0} G \cosh k_{0} h}{\delta_{0} \triangle\left(i k_{0}\right)},
$$

where

$$
\delta_{0}=2 k_{0} h+\sinh 2 k_{0} h, \quad \delta_{n}=2 k_{n} h+\sinh 2 k_{n} h,
$$




$$
\begin{aligned}
& a_{0}=\frac{1}{\pi \cosh k_{0} h} \int_{0}^{h} U(y) \cosh k_{0} h d y, \\
& a_{n}=-\frac{1}{\pi \cos k_{n} h} \int_{0}^{h} U(y) \cos k_{n} h d y
\end{aligned}
$$

Consequently

$$
\begin{gathered}
\phi_{1}=-8 e^{-\imath \pi v / 2} \sum_{n=1}^{\infty} \frac{a_{n} k_{n} \cos k_{n} h}{\delta_{n} \triangle\left(i k_{n}\right)}\left[b K_{v}^{\prime 2}\left(k_{n} b\right) I_{v}\left(k_{n} r\right)-\left[i G+b K_{v}^{\prime}\left(k_{n} b\right) \times\right.\right. \\
\left.\left.I_{v}^{\prime}\left(k_{n} b\right)\right] K_{v}\left(k_{n} r\right)\right] \cos k_{n}(h-y) \\
+4 \pi \frac{a_{0} k_{0} \cosh k_{0} h}{\delta_{0} \triangle\left(k_{0}\right)}\left[\frac{1}{2} \pi b\left(H_{v}^{(1)}\left(k_{0} b\right)\right)^{2} J_{v}\left(k_{0} r\right)-\left[G+\frac{1}{2} \pi b H_{v}^{(1) \prime}\left(k_{0} b\right) \times\right.\right. \\
\left.\left.J_{v}^{\prime}\left(k_{0} b\right)\right] H_{v}^{(1)}\left(k_{0} r\right)\right] \cosh k_{0}(h-y) \\
\phi_{2}=8 i G e^{-\imath \pi v / 2} \sum_{n=1}^{\infty} \frac{a_{n} k_{n} \cos k_{n} h}{\delta_{n} \triangle\left(i k_{n}\right)} K_{v}\left(k_{n} r\right) \cos k_{n}(h-y) \\
+4 \pi G \frac{a_{0} k_{0} \cosh k_{0} h}{\delta_{0} \triangle\left(k_{0}\right)} H_{v}^{(1)}\left(k_{0} r\right) \cosh k_{0}(h-y)
\end{gathered}
$$

We consider the following two special cases.

(i) When $U(y)=V$, $(V$ is a constant $)$. In this case

$$
a_{0}=-\frac{V}{\pi k_{0}} \tanh k_{0} h, \quad a_{n}=-\frac{V}{\pi k_{n}} \tanh k_{n} h
$$

Therefore

$$
\begin{gathered}
\phi_{1}=\frac{8 V}{\pi} e^{-\imath \pi v / 2} \sum_{n=1}^{\infty} \frac{\sin k_{n} h}{\delta_{n} \triangle\left(i k_{n}\right)}\left[b K_{v}^{\prime 2}\left(k_{n} b\right) I_{v}\left(k_{n} r\right)-\left[i G+b K_{v}^{\prime}\left(k_{n} b\right) \times\right.\right. \\
\left.\left.I_{v}^{\prime}\left(k_{n} b\right)\right] K_{v}\left(k_{n} r\right)\right] \cos k_{n}(h-y) \\
-4 V \frac{\sinh k_{0} h}{\delta_{0} \triangle\left(k_{0}\right)}\left[\frac{1}{2} \pi b\left(H_{v}^{(1)}\left(k_{0} b\right)\right)^{2} J_{v}\left(k_{0} r\right)-\left[G+\frac{1}{2} \pi b H_{v}^{(1) \prime}\left(k_{0} b\right) \times\right.\right. \\
\left.\left.J_{v}^{\prime}\left(k_{0} b\right)\right] H_{v}^{(1)}\left(k_{0} r\right)\right] \cosh k_{0}(h-y), \\
\phi_{2}=\frac{8 i V G}{\pi} e^{-\imath \pi v / 2} \sum_{n=1}^{\infty} \frac{\sin k_{n} h}{\delta_{n} \triangle\left(i k_{n}\right)} K_{v}\left(k_{n} r\right) \cos k_{n}(h-y) \\
+4 V G \frac{\sinh k_{0} h}{\delta_{0} \triangle\left(k_{0}\right)} H_{v}^{(1)}\left(k_{0} b\right) \cosh k_{0}(h-y),
\end{gathered}
$$

(ii) When $U(y)=V \cosh k_{0}(h-y)$, we get

$$
a_{0}=-\frac{\delta_{0} V}{4 \pi k_{0} \cosh k_{0} h}, \quad a_{n}=0,
$$

and 


$$
\begin{gathered}
\phi_{1}=-\frac{V}{\triangle\left(k_{0}\right)}\left[\frac{1}{2} \pi b\left(H_{v}^{(1)}\left(k_{0} b\right)\right)^{2} J_{v}\left(k_{0} r\right)-\left[G+\frac{1}{2} \pi b H_{v}^{(1) \prime}\left(k_{0} b\right) J_{v}^{\prime}\left(k_{0} b\right)\right] \times\right. \\
\left.H_{v}^{(1)}\left(k_{0} r\right)\right] \cosh k_{0}(h-y), \\
\phi_{2}=\frac{V G}{\triangle\left(k_{0}\right)} H_{v}^{(1)}\left(k_{0} r\right) \cosh k_{0}(h-y),
\end{gathered}
$$

\section{OBLIQUE WAVES GENERATED BY THE POROUS WALL}

If we now let the porous wall oscillate obliquely with velocity $U(y) \exp (-i \omega t+i v \theta)$ while the impermeable wall at $r=a$ be kept fixed, then the new boundary value problem is the same as stated in Section 2 except that the boundary conditions (23), (2.5) are replaced by

$$
\begin{gathered}
\frac{\partial}{\partial r} \phi_{1}=0, \quad \text { on } \quad r=a \\
\frac{\partial}{\partial r} \phi_{\jmath}-U(y)=i G\left(\phi_{1}-\phi_{2}\right), \quad \text { on } \quad r=b
\end{gathered}
$$

Thus when the porous wall is the wave generator we have

$$
\begin{aligned}
\phi_{1}= & \frac{4 b}{\pi} e^{-\imath \pi v / 2} \int_{0}^{\infty} \frac{k a(k) f(k, y)}{\left(k^{2}+K^{2}\right) \triangle(i k)}\left[I_{v}(k r) K_{v}^{\prime}(k a)-K_{v}(k r) I_{v}^{\prime}(k a)\right] K_{v}^{\prime}(k b) d k \\
& -\frac{\pi^{2} b K A}{\triangle(K)}\left[J_{v}(k r) H_{v}^{(1) \prime}(K a)-H_{v}^{(1)}(K r) J_{v}^{\prime}(K a)\right] H_{v}^{(1) \prime}(K b) e^{-K y} \\
\phi_{2}= & \frac{4 b}{\pi} e^{-\imath \pi v / 2} \int_{0}^{\infty} \frac{k a(k) f(k, y)}{\left(k^{2}+K^{2}\right) \triangle(i k)}\left[I_{v}(k b) K_{v}^{\prime}(k a)-K_{v}^{\prime}(k b) I_{v}^{\prime}(k a)\right] K_{v}(k r) d k \\
& -\frac{\pi^{2} b K A}{\triangle(K)}\left[J_{v}^{\prime}(K b) H_{v}^{(1) \prime}(K a)-H_{v}^{(1) \prime}(K b) J_{v}^{\prime}(k a)\right] H_{v}^{(1)}(K r) e^{-K y} .
\end{aligned}
$$

When $M=0$, the waves are trapped in the bounded region between the two cylinders $a \leq r \leq b$ and no waves radiate away from the wall, liquid simply piles up around the wall.

\section{WAVE TRAPPING}

In this section we investigate an interesting application of the above results to the case of a time cylindrical wave $C H_{v}^{(2)}(k R) \exp (i v \theta-K y)$ incident obliquely, proceeding from infinity, the porous cylindrical wall at $r=b$ and the impermeable cylindrical wall at $r=a$ both fixed. The velocity potentials $\phi_{J}(r, y)$ are functions that satisfy $(2.1),(2.2)$ and (2.6). On the porous wall

$$
\left.\begin{array}{rl}
\frac{\partial}{\partial r} \phi_{1} & =\frac{\partial}{\partial r} \phi_{2} \\
& =i G\left(\phi_{1}-\phi_{2}\right),
\end{array}\right\} r=b
$$

and on the impermeable wall

$$
\frac{\partial}{\partial r} \phi_{1}=0 \quad \text { on } \quad r=a
$$

Moreover

$$
\phi_{2} \rightarrow C H_{v}^{(2)}(K r) e^{-K y}+A H_{v}^{(1)}(K r) e^{-K y}, \quad \text { as } \quad r \rightarrow \infty
$$


Here $A$ (to be determined) is a complex constant relating to the amplitude and phase of the reflected wave

Consider the functions

$$
\Psi_{J}=\phi_{J}(r, y)-2 C J_{v}(K r) e^{-K y}
$$

These new functions satisfy equations ( 21$)$ and the free surface boundary conditions On the porous wall $(r=b)$,

$$
\begin{aligned}
\frac{\partial}{\partial r} \Psi_{1} & =\frac{\partial}{\partial r} \Psi_{2} \\
& =i G\left(\Psi_{1}-\Psi_{2}\right)-2 C J_{v}^{\prime}(K b) e^{-K y},
\end{aligned}
$$

and on the impermeable wall

$$
\frac{\partial}{\partial r} \Psi_{1}=-2 C J_{v}^{\prime}(K a) e^{-K y}
$$

And

$$
\Psi_{2} \rightarrow(A-C) H_{v}^{(1)}(K r) e^{-K y}, \quad \text { as } \quad r \rightarrow \infty .
$$

Since the present problem is linear, $\Psi_{1}, \Psi_{2}$ can be obtained by a suitable superposition of the results (3 7), (5.3) and (3.8), (5 4) respectively. Hence

$$
\begin{gathered}
\phi_{1}=\frac{2 C G}{\triangle(K)}\left[J_{v}(K r) H_{v}^{(1) \prime}(K a)-J_{v}^{\prime}(K a) H_{v}^{(1)}(K r)\right] e^{-K y} \\
\phi_{2}=\left[-C \frac{\triangle *(K)}{\triangle(K)} H_{v}^{(1)}(K r)+C H_{v}^{(2)}(K r)\right] e^{-K y}
\end{gathered}
$$

where $\triangle^{*}(K)=G H_{v}^{(2) \prime}(K a)+M(K) H_{v}^{(2) \prime}(K b)$.

The coefficient of reflection $R$ is defined as the square of the ratio of the amplitude of the reflected wave to the amplitude to the incident wave i.e.

$$
R=\left|\frac{\triangle^{*}(K)}{\triangle(K)}\right|=\frac{\alpha^{2}-2 M^{2} G+\beta^{2} M^{2}}{\alpha^{2}+2 M^{2} G+\beta^{2} M^{2}}
$$

where $\alpha^{2}=\frac{1}{2} \pi b\left[J_{v}^{\prime 2}(K a)+Y_{v}^{\prime 2}(K a)\right], \beta^{2}=\frac{1}{2} \pi b\left[J_{v}^{\prime 2}(K b)+Y_{v}^{\prime 2}(K b)\right]$ when the wall at $r=b$ is impermeable i.e., when $G=0$, the incident wave is totally reflected by it. We get the same situation when the wall $(r=b)$ is completely permeable but now the wave is totally reflected by the impermeable wall at $r=a$. We note also that when $M=0$ i.e. when $a$ and $b$ has values satisfying the equation

$$
J_{v}^{\prime}(K b) Y_{v}^{\prime}(K a)-J_{v}^{\prime}(K a) Y_{v}^{\prime}(K b)=0,
$$

the incident wave is totally reflected $(R=1)$ at $r=b$ irrespective of the value of $G$. By simple differentiation of (6.11) with respect to $G$ for any fixed values of $a$ and $b, R$ reduces to a minimum,

$$
R_{\min }=\frac{\alpha \beta-M}{\alpha \beta+M},
$$

when $G=\frac{M \beta}{\alpha}$; this minimum value vanishes when $\alpha \beta=M$ i.e., when $a$ and $b$ satisfy the equation

$$
J_{v}^{\prime}(K b) J_{v}^{\prime}(K a)+Y_{\imath}^{\prime}(K b) Y_{v}^{\prime}(K a)=0,
$$

That is $R=0$ when $G=\beta^{2}$ and $a, b$ has values satisfying equation (6.14). Under these circumstances the porous wall acts as an efficient wave absorber or eliminator for the incident waves, i.e., for $G=\beta^{2}$ 
and for values of $a$ and $b$ which satisfy equation (614), there is a wave trapping phenomenon, that is waves will be trapped inside the region $a \leq r \leq b$

Now we give an estimate for $R_{\min }$ (equation (613)) for large $K a$ and $K b$ for the case of radial incident waves $(v=0)$ This can be done using the asymptotic formulas of $J_{1}(K r)$ and $Y_{1}(K r)$ for larger $K r$ which are

$$
J_{1}(K r) \sim \sqrt{\frac{2}{\pi K r}} \sin \left(K r-\frac{\pi}{4}\right), \quad Y_{1}(K r) \sim-\sqrt{\frac{2}{\pi K r}} \cos \left(K r-\frac{\pi}{4}\right) .
$$

In this case

$$
\frac{\alpha^{2}}{K} \simeq \frac{b}{a}, \quad \frac{\beta^{2}}{K} \simeq 1, \quad \frac{M}{K} \simeq \frac{b}{a} \sin K(b-a)
$$

Thus

$$
R_{\min } \simeq \frac{1-\sin K(b-a)}{1+\sin K(b-a)}
$$

and therefore, $R_{\min } \sim 0$ when $K(b-a)=\frac{\pi}{2}+\pi s, s=0,1,2, \ldots$. This means the porous wall together with the fluid region between it and the impermeable wall acts as an efficient wave absorber for incident waves of wave length $\frac{4(b-a)}{1+2 s}$. In fact this result agrees with that obtained in the two dimensional surface wave case treated in [13].

\section{NUMERICAL RESULTS}

For radial incident waves $(v=0)$, since

$$
J_{0}^{\prime}(K r)=-K J_{1}(K r) \quad \text { and } \quad Y_{0}^{\prime}(K r)=-K Y_{1}(K r),
$$

the conditions (6.14) for wave trapping and (6.12) for total reflection become

$$
\begin{aligned}
& J_{1}(K b) J_{1}(K a)+Y_{1}(K b) Y_{1}(K a)=0, \\
& J_{1}(K b) J_{1}(K a)-Y_{1}(K b) Y_{1}(K a)=0,
\end{aligned}
$$

respectively For those values of $K a$ and $K b$ which satisfy (7.1), $\frac{G}{K}=\frac{1}{2} \pi b K\left[J_{1}^{2}(K b)+Y_{1}^{2}(K b)\right]$. When the angle of incident waves makes $30^{\circ}$ with the radial direction $(v=0.5)$, since

$$
J_{1 / 2}(K r)=\sqrt{\frac{2}{\pi K r}} \sin K r, \quad Y_{1 / 2}(K r)=\sqrt{\frac{2}{\pi K r}} \cos K r,
$$

conditions (6.14) and (6.12) now take the simpler forms

$$
\begin{aligned}
& \left(4 a b K^{2}+1\right) \cos K(b-a)+2 K(b-a) \sin K(b-a)=0, \\
& 2 K(b-a) \cos K(b-a)-\left(4 a b K^{2}+1\right) \sin K(b-a)=0 .
\end{aligned}
$$

Thus for the case of $v=0.5$ and those values of $K a, K b$ which satisfy (6.3), $\frac{G}{K}=1+\frac{1}{4 K^{2} b^{2}}$. For fixed $K a$ or $K b$ equations (7.1)-(7.4) has infinite number of real roots. Table 1 lists the first few roots $K b(>K a)$ of equations (7.1), (7.3) which correspond $v=0$ and $v=0.05$ respectively for fixed $K a=2 \pi$ and the corresponding value of $\frac{G}{K}$ at each $K b$ Table 2 lists also the first few roots of (7.2), (7.4) for $K a=2 \pi$ and $\frac{\pi}{2}$. We note that for all cases listed successive large roots differ approximately by $\pi$ and that $\frac{G}{K} \simeq 1$. 
Table 1. Values of $K b(>K a)$ and $G / K$ for wave trapping

\begin{tabular}{|c|c|c|c|}
\hline$v=0$ & $G / K$ & $v=0.5$ & $G / K$ \\
\hline 786568689 & 100597502 & 651475569 & 100589039 \\
\hline 1102072268 & 1.00306449 & 968134717 & 1.00266728 \\
\hline 1416981407 & 100185914 & 1283560523 & 100151743 \\
\hline 1731618973 & 100124677 & 1598486294 & 100097841 \\
\hline 2046109864 & 100089374 & 1913159616 & 100068303 \\
\hline 2360512575 & 100067188 & 2227677662 & 100050377 \\
\hline 2674858147 & 100052344 & 25.42124425 & 1.00038685 \\
\hline 2989164583 & 1.00041925 & 2856500080 & 1.00030639 \\
\hline 33.03443042 & 100034334 & 31.70832819 & 1.00024865 \\
\hline 3617700808 & 100028632 & 3485134260 & 1.00020583 \\
\hline 3931942840 & 100024241 & 3799412174 & 1.00017138 \\
\hline 42.46172629 & 100020788 & 41.13671958 & 1.00014773 \\
\hline 4560392703 & 1.00018023 & 44.27917471 & 1.00012751 \\
\hline 4874604942 & 1.00015775 & 47.42151553 & 100011117 \\
\hline 51.88810767 & 100013923 & 5056376335 & 1.00009778 \\
\hline
\end{tabular}

Table 2 Values of $K b(>K a)$ for complete reflection

\begin{tabular}{|r|r|r|r|}
\hline \multicolumn{2}{|c|}{$v=0$} & \multicolumn{2}{c|}{$v=0.5$} \\
\hline$K a=2 \pi$ & $K a=\pi / 2$ & \multicolumn{1}{|c|}{$K a=2 \pi$} & $K a=\pi / 2$ \\
\hline 9.44431648 & 4.84806631 & 945133478 & 4.91926471 \\
\hline 12.59573441 & 8.01922751 & 1260613831 & 8.10050436 \\
\hline 15.74324000 & 1117382989 & 1575564940 & 11.25936504 \\
\hline 18.88878187 & 14.32274063 & 18.90252072 & 14.41065337 \\
\hline 22.03319888 & 17.46902417 & 22.04788468 & 17.55846006 \\
\hline 25.17691168 & 20.61387985 & 25.19230664 & 20.70437653 \\
\hline 28.320154444 & 23.75787344 & 28.33610051 & 23.84915194 \\
\hline 31.46306786 & 26.90130671 & 31.47945463 & 26.99318555 \\
\hline 34.60574159 & 30.04435529 & 34.62248884 & 30.13670974 \\
\hline 37.74823545 & 33.18712837 & 37.76528307 & 33.27986897 \\
\hline 40.89059088 & 3632969739 & 40.90789266 & 36.42275777 \\
\hline 4403283750 & 39.47211105 & 4405035712 & 39.56544062 \\
\hline 47.17499704 & 42.61440369 & 4719270547 & 42.70796301 \\
\hline 50.31708581 & 45.75660024 & 50.33495946 & 45.85035794 \\
\hline 53.45911628 & 48.89871921 & 53471352 & 48.99264994 \\
\hline
\end{tabular}




\section{REFERENCES}

[1] FAULKNER, T R, Diffraction of an obliquely incident surface wave by a vertical barrier of finite depth, Proc. Camb. Philos. Soc. 62 (1966), 829-838

[2] FAULKNER, T R, Diffraction of an obliquely incident surface wave by a submerged plane barrier, Z. Angew. Math. Phys. 17 (1966), 699-707.

[3] JARVIS, R J and TAYLOR, B S., The scattering of surface waves by a vertical plane barrier, Proc. Camb. Soc. 66 (1969), 417-422.

[4] EVANS, D.V. and MORRIS, C.A N., The effect of a fixed vertical barrier on obliquely incident surface waves in deep water, J. Inst. Math. Appl. 9 (1972), 198-204.

[5] RHODES-ROBINSON, P.F., Note on the reflection of water waves at a wall in the presence of surface tension, Proc. Camb. Philos. Soc. 92 (1982), 369-373

[6] MANDEL, B N. and GOSWAMI, S K., A note on the diffraction of an obliquely incident surface wave by a partially immersed fixed vertical barrier, App. Sci. Res. 40 (1983), 345-353.

[7] LEVINE, H., Scattering of surface waves by a submerged circular cylinder, J. Math. Phys. 6 (1965), 1231-1243.

[8] GARRISON, C J., On the interaction of an infinite shallow draft cylinder oscillating at the free surface with a train of oblique waves, J. Fluid Mech 39 (1969), 227-255

[9] BAI, K.J., Diffraction of oblique waves by an infinite cylinder, J. Fluid Mech. 68 (1975), 513-535.

[10] CHWANG, A.T., A porous wave maker theory, J. Fluid Mech. 132 (1983), 395-406.

[11] CHWANG, A.T. and LI, W., A piston-type porous wavemaker theory, J. Eng. Math. 17 (1983), 301-313.

[12] CHWANG, A.T. and DONG, Z., Wave-trapping due to a porous plate, Proc. 15th Symposium on Naval Hydrodynamics, pp. 407-417, Washington: National Academy Press, 1985.

[13] GORGUI, M.A. and FALTAS, M.S., Forced surface waves in the presence of porous walls, Acta Mechanica 79 (1989), 259-275.

[14] TAYLOR, G.I., Fluid flow in regions bounded by porous surfaces, Proc. R. Soc. Lond. A234 (1956), 456-475. 


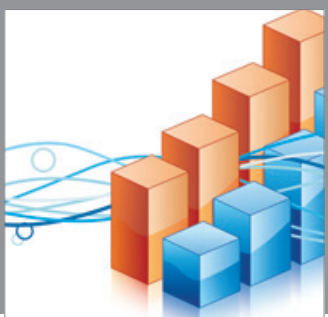

Advances in

Operations Research

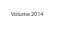

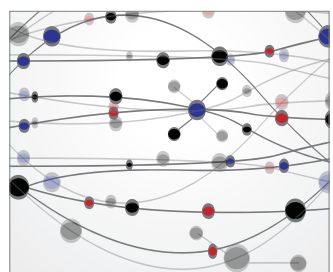

\section{The Scientific} World Journal
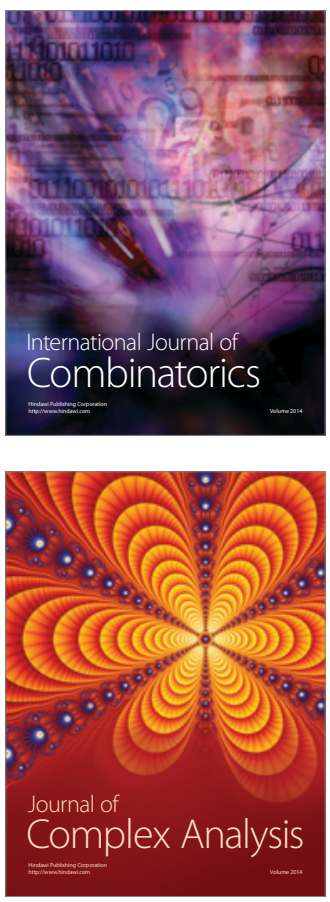

International Journal of

Mathematics and

Mathematical

Sciences
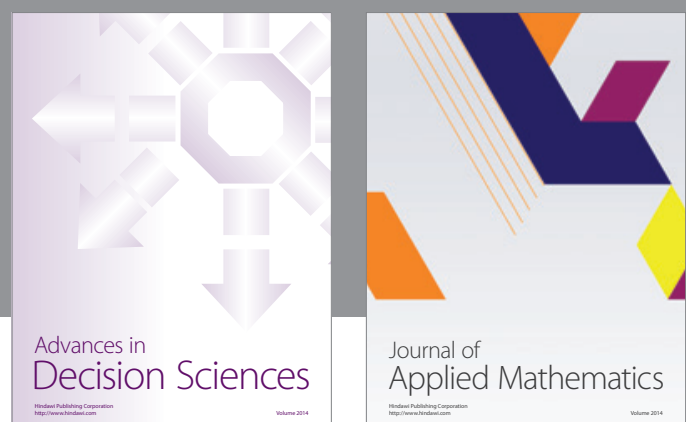

Journal of

Applied Mathematics
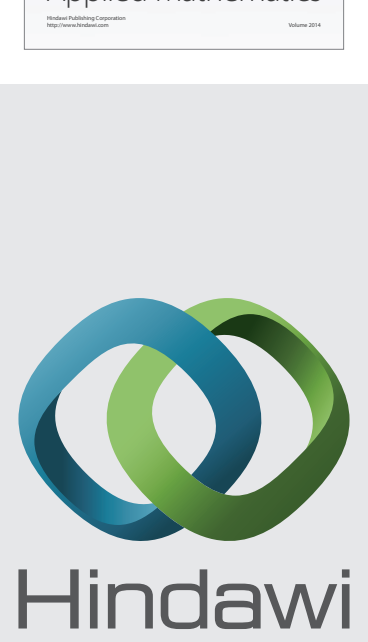

Submit your manuscripts at http://www.hindawi.com
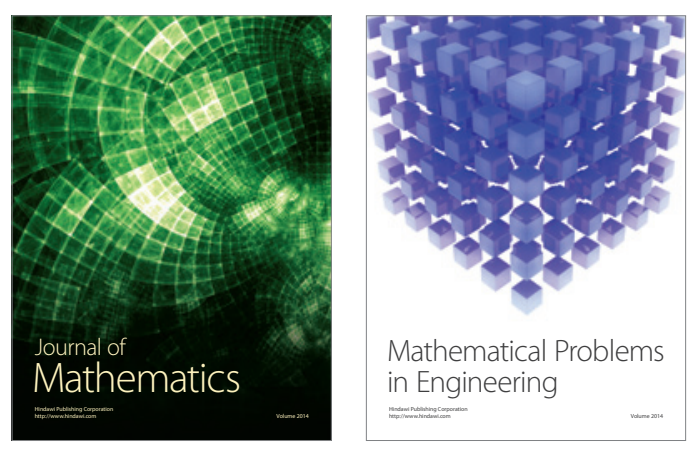

Mathematical Problems in Engineering
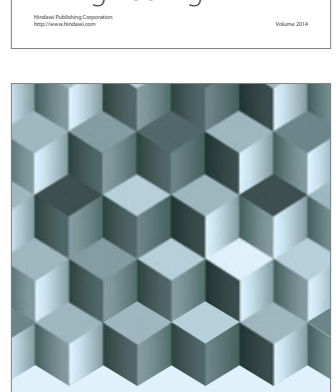

Journal of

Function Spaces
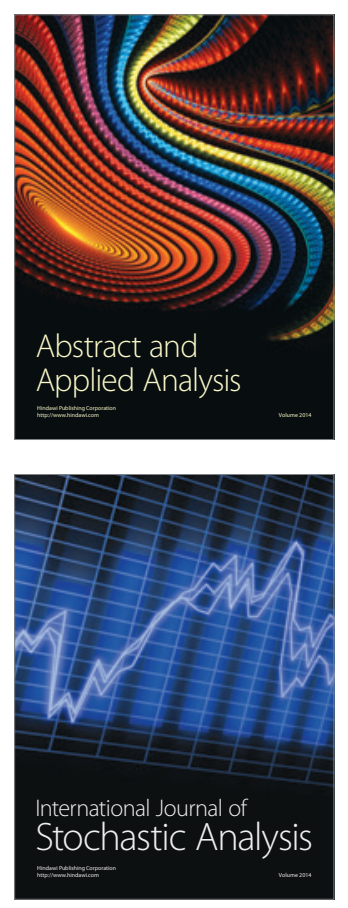

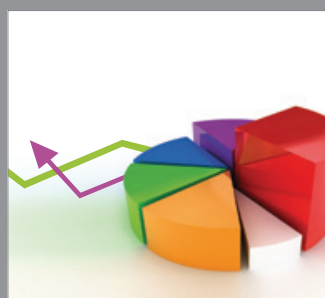

ournal of

Probability and Statistics

Promensencen
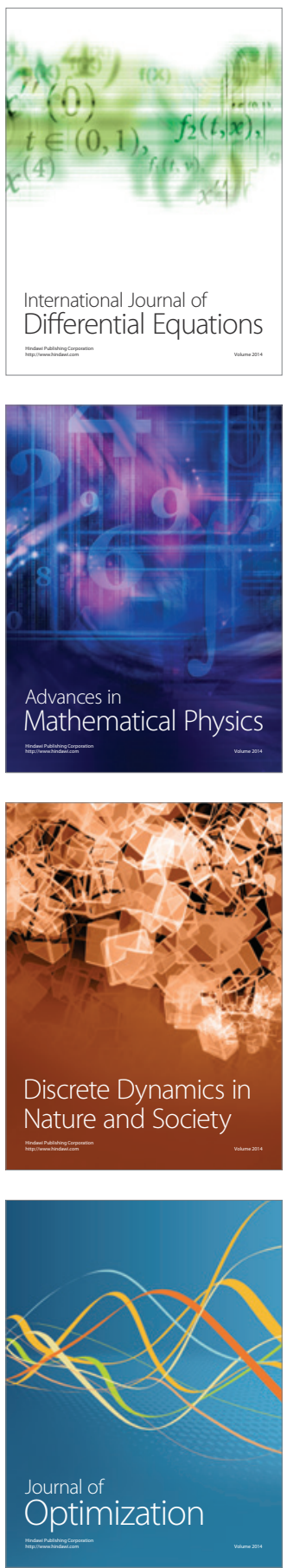